This item was submitted to Loughborough's Research Repository by the author.

Items in Figshare are protected by copyright, with all rights reserved, unless otherwise indicated.

\title{
Water-wave propagation through an infinite array of cylindrical structures
}

PLEASE CITE THE PUBLISHED VERSION

LICENCE

CC BY-NC-ND 4.0

\section{REPOSITORY RECORD}

Mclver, P.. 2019. "Water-wave Propagation Through an Infinite Array of Cylindrical Structures". figshare. https://hdl.handle.net/2134/837. 


\title{
Water-wave propagation through an infinite array of cylindrical structures
}

\author{
P. McIver \\ Department of Mathematical Sciences, Loughborough University, \\ Loughborough, Leics, LE11 3TU, UK
}

\begin{abstract}
An investigation is made into water-wave propagation through a doubly-periodic array of vertical cylinders extending to infinity in both horizontal directions. Methods are presented for the calculation of the frequency ranges for which wave propagation without change of amplitude is possible ('passing bands'), and for which propagation without change of amplitude is not possible ('stopping bands'). Some of the techniques may be used to determine the decay of wave amplitude within the stopping bands. Approximate and numerical techniques are used to show how this infinite-array problem is related to trapped modes, Rayleigh-Bloch waves, and the problem of wave diffraction by a grating made up of a finite number of cylinder rows.
\end{abstract}




\section{Introduction}

The problem of a particle moving in a periodic potential has been studied extensively in solid-state physics and there is an established formalism for its investigation (Ashcroft \& Mermin, 1976). A mathematically related problem is the propagation of water waves above a patch of sea bed with undulations that are periodic in one horizontal direction (see O'Hare \& Davies, 1993, and references therein). Given a particular sea bed, it is observed that there are ranges of frequency where there is strong reflection, and complementary ranges for which there is weak reflection of a monochromatic incident wave. Recently, Chou (1998) has considered water-wave interaction with an infinite array of periodically arranged surface scatterers using the formalism of solid state physics. He investigated geometries with finite periodicity in both one and two horizontal directions and found that for certain ranges of frequency wave propagation through the array without change in amplitude is possible, although in general there will be a change in phase from one scatterer to another. In complementary ranges of frequency, wave propagation without change of amplitude is not possible. In the terminology of solid-state physics, these ranges of frequency are known as passing bands and stopping bands, respectively.

In this paper, the propagation of waves through a doubly periodic array of identical vertical cylinders is examined in detail. This work is motivated in part by a proposal for a airport in Japan that involves a floating platform supported by thousands of cylindrical legs. Over recent years, this has provoked interest in how water waves interact with very large arrays of vertical cylinders (Kagemoto \& Yue, 1986; Kagemoto, 1998; Kashiwagi, 1999). The main purpose of the work is to investigate how knowledge of the relatively straightforward problem of wave propagation through infinite arrays of cylinders can be used to make deductions about propagation through finite arrays. Although set into the context of water waves, the present work also has an acoustic interpretation. Related work on sound propagation through tube bundles is reported by Heckl \& Mulholland (1995).

The problem is formulated in $\S 2$ in terms of a velocity potential for the flow. Because of the doubly-periodic arrangement of cylinders, it is possible to confine attention to a cell in a horizontal plane that contains only a single cylinder. For a rectangular array this primitive cell is also rectangular and so-called Bloch conditions are used to relate the potential and its normal derivative on opposite sides of the rectangle. Throughout this paper the problem of wave propagation through an infinite array will be referred to as 'the Bloch problem'. An approximate solution of the Bloch problem for unidirectional wave propagation is presented in $\S 3$. This is used to show how passing and stopping bands arise in the water-wave problem. Within a passing band waves are able to propagate through the array without change of amplitude. Within stopping bands there is an 
exponential decrease of wave amplitude with distance.

For a passing band the boundary-value problem is self-adjoint and the frequencies can be calculated quite straightforwardly by means of a variational principle. This method is used in $\S 4$ to investigate how the band structure develops as the cylinder radius is increased from zero. In addition, calculations are presented to show how the solutions obtained approach previously-known trapped mode and Rayleigh-Bloch wave solutions as one of the cell dimensions is allowed to increase without bound. In this context, the term Rayleigh-Bloch wave is used to describe a non-periodic wave that propagates along an infinitely-long line of equally-spaced cylinders and decays to zero in the perpendicular horizontal direction. The term trapped mode is here reserved for certain limiting cases when the solution and the geometry have the same periodicity.

The variational method of $\S 4$ gives no information about the rate of amplitude decay within stopping bands. Two numerical methods which calculate this decay are given in $\S 5$. In the first method, a series that satisfies the cylinder boundary condition identically is applied pointwise to satisfy the conditions on the cell walls. This method is straightforward to use but is not very robust for higher frequencies. The second technique is a more robust, but also more computationally expensive, boundary-integral method based on an application of Green's theorem.

The final sections of the paper deal with wave diffraction by a finite number of parallel rows of cylinders, where each row is of infinite length. In $\S 6$, a wide-spacing formulation is used to demonstrate explicitly how, for the case of wave propagation normal to the rows, the transmission beyond a finite number of rows is related to the Bloch problem. In $\S 7$, various numerical calculations using the methods of $\S \S 4-5.2$ are used to investigate the finite-array problem when waves are able to propagate in more than one direction.

\section{General formulation}

A periodic array of identical, rigid, circular cylinders, which extend to infinity in all horizontal directions, stands in water of constant depth $h$. Cartesian coordinates are chosen so that the $x$ and $y$ axes lie in a horizontal plane and the $z$ axis is directed vertically upwards. The origin of coordinates is on the axis of one of the cylinders and located in the plane of the mean free surface. Water waves of radian frequency $\omega$ propagate through the array. The water is assumed to be inviscid and incompressible and the flow to be irrotational. The cylinders extend throughout the depth and so, under the usual assumptions of the linearised theory of water waves, solutions for the velocity potential may be sought in the form

$$
\Phi(x, y, z, t)=\operatorname{Re}\left\{\phi(x, y) \cosh \kappa(z+h) \mathrm{e}^{-\mathrm{i} \omega t}\right\}
$$


where

$$
\left(\nabla^{2}+\kappa^{2}\right) \phi=0
$$

throughout the fluid region and $\kappa$ is the real positive root of the dispersion relation

$$
\omega^{2}=g \kappa \tanh \kappa h .
$$

The geometrical description of the array (or lattice) of cylinders adopted here is that used in the theory of crystal structures in solid state physics (see Ashcroft \& Mermin, 1976, Chapters 4 and 5). Let $\mathbf{a}_{1}$ and $\mathbf{a}_{2}$ be two vectors that span the lattice, that is every translation between the axes of cylinders in the horizontal plane has the form of a so-called lattice vector

$$
\mathbf{R}=m_{1} \mathbf{a}_{1}+m_{2} \mathbf{a}_{2},
$$

where $m_{1}$ and $m_{2}$ are integers. It is also convenient to introduce so-called reciprocal lattice vectors K satisfying

$$
\mathbf{K} \cdot \mathbf{R}=2 \pi p
$$

where $p$ is an integer. If the reciprocal lattice vectors are expressed in the form

$$
\mathbf{K}=n_{1} \mathbf{b}_{1}+n_{2} \mathbf{b}_{2}
$$

for integers $n_{1}, n_{2}$ then (5) is satisfied provided

$$
\mathbf{a}_{i} \cdot \mathbf{b}_{j}=2 \pi \delta_{i j},
$$

where $\delta_{i j}$ is the Kronecker delta.

In other contexts (Ashcroft \& Mermin, 1976, Chapter 8; Chou, 1998), Bloch's theorem is used to justify looking for solutions in the form

$$
\phi(\mathbf{r})=\mathrm{e}^{\mathrm{iq} \cdot \mathbf{r}} \psi(\mathbf{r}),
$$

where $\mathbf{r}$ is the position vector of an arbitrary point in the array, $\mathbf{q}$ is a real-valued vector, and the function $\psi$ has the same periodicity as the lattice, that is

$$
\psi(\mathbf{r}+\mathbf{R})=\psi(\mathbf{r})
$$

for all lattice vectors $\mathbf{R}$. Here solutions are also sought in this form, but in the water-wave problem q may be complex. The above is exactly equivalent to seeking solutions that satisfy

$$
\phi(\mathbf{r}+\mathbf{R})=\mathrm{e}^{\mathrm{i} \mathbf{q} \cdot \mathbf{R}} \phi(\mathbf{r})
$$




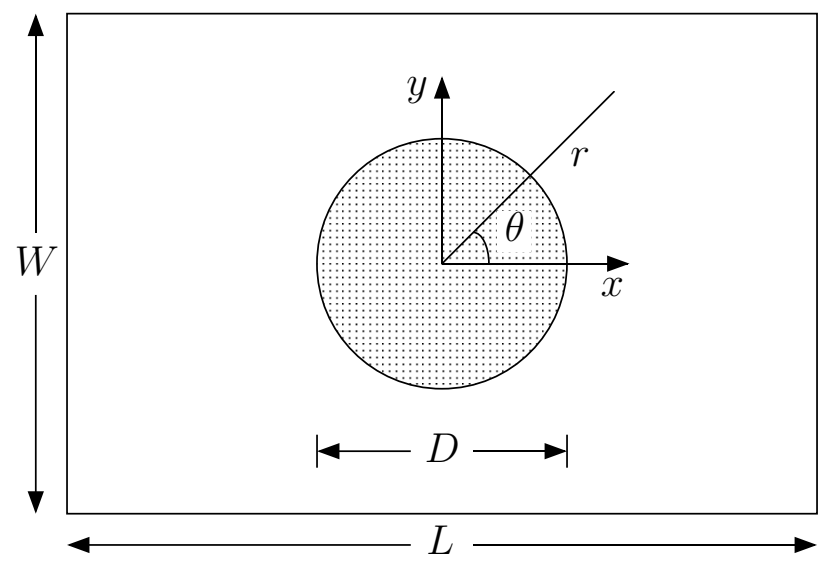

Figure 1: Definition sketch for one cell of a rectangular array.

where $\mathbf{R}$ is again any lattice vector. If $\mathbf{q}$ is real then it measures the change in the phase of the motion as the lattice is traversed. If $\mathbf{q}$ has a non-zero imaginary part then there is also a decay in amplitude as a wave propagates through the array.

Two basic approaches to the problem are used here. One approach is to specify the wave vector $\mathbf{q}=q_{1} \mathbf{i}+q_{2} \mathbf{j}$ and then solve for the wavenumber $\kappa$ (here $\mathbf{i}$ and $\mathbf{j}$ are unit vectors in the $x$ and $y$ directions, respectively). Alternatively, $\kappa$ and one component of $\mathbf{q}$ is specified and the problem is then to determine the second component of $\mathbf{q}$.

Equation (10) is unchanged if $\mathbf{q}$ is augmented by any reciprocal lattice vector $\mathbf{K}$. Thus, given a solution $\phi(\mathbf{r} ; \mathbf{q})$ then $\phi(\mathbf{r} ; \mathbf{q}+\mathbf{K})$ is also a solution. Consequently, it is sufficient to restrict attention to the so-called 'first Brillouin zone' $\left\{\operatorname{Re} q_{1} L \in[-\pi, \pi], \operatorname{Re} q_{2} W \in[-\pi, \pi]\right\}$, as long as it is born in mind that for any pair $(\kappa, \mathbf{q})$, for which the problem has a non-trivial solution, such solutions also exist for pairs $(\kappa, \mathbf{q}+\mathbf{K})$. Further, if any component of $\mathbf{q}$ is reversed in sign this corresponds to a reversal of the direction of propagation of that component. Thus, attention is confined to the region $\left\{\operatorname{Re} q_{1} L \in[0, \pi], \operatorname{Re} q_{2} W \in[0, \pi]\right\}$ and all results for $\mathbf{q}$ will be given within this region. This method of displaying results is known as a 'reduced zone scheme' (Ashcroft \& Mermin, 1976, p. 160).

For a rectangular array of cylinders of diameter $D$ with

$$
\mathbf{a}_{1}=L \mathbf{i} \quad \text { and } \quad \mathbf{a}_{2}=W \mathbf{j}
$$

it is sufficient to consider a single rectangular cell of length $L$ and width $W$ as illustrated in figure 1 . The corresponding primitive reciprocal lattice vectors are

$$
\mathbf{b}_{1}=\frac{2 \pi \mathbf{i}}{L} \quad \text { and } \quad \mathbf{b}_{2}=\frac{2 \pi \mathbf{j}}{W} .
$$


For this geometry, equation (10) is equivalent to the four independent conditions

$$
\left.\begin{array}{l}
\phi(L / 2, y)=\mathrm{e}^{\mathrm{i} q_{1} L} \phi(-L / 2, y) \\
\frac{\partial \phi}{\partial x}(L / 2, y)=\mathrm{e}^{\mathrm{i} q_{1} L} \frac{\partial \phi}{\partial x}(-L / 2, y)
\end{array}\right\}, \quad|y| \leq W / 2,
$$

The mathematical problem has been reduced to the solution the field equation (2) within the fluid region of the cell $\{|x| \leq L / 2,|y| \leq W / 2\}$ subject to the boundary conditions (13)-(14) and the condition of no flow through the cylinder wall, namely

$$
\frac{\partial \phi}{\partial r}=0 \quad \text { on } \quad r=D / 2,
$$

where $(r, \theta)$ are standard plane polar coordinates with origin at the centre of the primitive cell illustrated in figure 1.

\section{An approximate solution}

To illustrate clearly some important features of the problem defined by equations (2) and (13)-(15) an approximate solution is now presented for the case $q_{2}=0$. The main assumption is that the cell length in the $x$ direction is much greater than the wavelength so that $\kappa L>>1$. Further, only solutions symmetric about $y=0$ are considered and the boundary conditions (14) are replaced by the special case

$$
\frac{\partial \phi}{\partial y}=0 \quad \text { on } \quad y= \pm W / 2,
$$

which is equivalent to having solid channel walls at $y= \pm W / 2$. The conditions (13) are retained in their general form. It will also be assumed that the wavenumber satisfies $\kappa W<2 \pi$ so that only waves with no $y$ dependence may propagate in a channel of width $W$. The main aim is to calculate the so-called Bloch transmission coefficient

$$
T_{\mathrm{B}}=\mathrm{e}^{\mathrm{i} q_{1} L}
$$

which measures the phase change and attenuation of a wave as it propagates through one cell of the array in the direction of $x$ increasing (see equations 13).

If the length $L$ of the cell is sufficiently large, then only plane waves propagating along the channel can exist in the vicinity of $x= \pm L / 2$, the evanescent modes will be negligible. Thus, in the neighbourhood of $x=-L / 2$,

$$
\phi=A_{1} \mathrm{e}^{\mathrm{i} \kappa x}+B_{1} \mathrm{e}^{-\mathrm{i} \kappa x}
$$


and in the neighbourhood of $x=L / 2$,

$$
\phi=A_{2} \mathrm{e}^{\mathrm{i} \kappa x}+B_{2} \mathrm{e}^{-\mathrm{i} \kappa x}
$$

for some complex constants $A_{1}, A_{2}, B_{1}$ and $B_{2}$. The wave with amplitude $A_{2}$ propagates away from the cylinder and is due to the transmission of $A_{1}$ past the cylinder and the reflection of $B_{2}$ from the cylinder. Similarly, the wave with amplitude $B_{1}$ arises from the transmission of $B_{2}$ and the reflection of $A_{1}$. Thus

$$
A_{2}=T A_{1}+R B_{2} \quad \text { and } \quad B_{1}=T B_{2}+R A_{1}
$$

where $R$ and $T$ are the reflection and transmission coefficients for a single cylinder in the channel (c.f. Heckl, 1992, equations 37). The boundary conditions (15) and (16) are used in the calculation of $R$ and $T$ which are assumed to be known for all $\kappa$. Application of the Bloch conditions (13) gives two further equations which, together with equations (20), yield a system which has a non-trivial solution provided $T_{\mathrm{B}}=\mathrm{e}^{\mathrm{i} q_{1} L}$ satisfies the quadratic

$$
T_{\mathrm{B}}^{2} T \mathrm{e}^{-\mathrm{i} \kappa L}-T_{\mathrm{B}}\left(T^{2}-R^{2}+\mathrm{e}^{-2 \mathrm{i} \kappa L}\right)+T \mathrm{e}^{-\mathrm{i} \kappa L}=0
$$

If the complex number $T$ is expressed in the form

$$
T=|T| \mathrm{e}^{\mathrm{i} \delta}
$$

then it may be shown that

$$
R=|R| \mathrm{e}^{\mathrm{i}(\delta \pm \pi / 2)}
$$

(Mei, 1983, $\S 7.6 .2$, describes the derivation for purely two-dimensional case, the channel case is a trivial extension). Use of these relations and the energy relation

$$
|R|^{2}+|T|^{2}=1
$$

valid below the cut-off wave number $\kappa W=2 \pi$, allows the above quadratic to be rewritten as

$$
T_{\mathrm{B}}^{2}-\frac{2 \cos (\delta+\kappa L)}{|T|} T_{\mathrm{B}}+1=0
$$

The product of the roots is unity so that if $\mathrm{e}^{\mathrm{i} q_{1} L}$ is a root then so is $\mathrm{e}^{-\mathrm{i} q_{1} L}$. The sum of the roots then yields

$$
\cos q_{1} L=\frac{\cos (\delta+\kappa L)}{|T|} \equiv f(\kappa L),
$$

say (c.f. Ashcroft \& Mermin, 1976, p. 148), which is an equation for $q_{1} L$ entirely in terms of real quantities. Provided $|f(\kappa L)| \leq 1$, equation (26) has only real solutions for $q_{1} L$ and waves will 
propagate through the array with their amplitude unchanged. The ranges of frequency for which this occurs are known in solid-state physics as 'passing bands'. However, whenever $|f(\kappa L)|>1$ solutions are of the form $q_{1} L=n \pi+\mathrm{i} Q$ for real $Q$ and some integer $n$. As the product of the roots is unity, the two roots have the form $(-1)^{n} \mathrm{e}^{\mp Q}$ with now $Q>0$. Waves that propagate in the direction of $x$ increasing correspond to the upper sign, while the lower sign corresponds to waves propagating in the direction of $x$ decreasing. In both cases the amplitude of the motion decreases as the wave propagates. The ranges of frequency for which this occurs are known in solid-state physics as 'stopping bands'.

In contrast to the application of this theory to crystal lattices, complex $\mathbf{q}$ in the water-wave problem has a physical interpretation. Further discussion of this is given later in $\S 5.1$ where a method for the computation of complex $\mathbf{q}$ is presented. The next section is devoted to a method of computation valid for real $\mathbf{q}$ only.

\section{Variational formulation of the eigenvalue problem}

\subsection{Method}

Attention is now turned to numerical methods for the calculation of solutions to the problem defined by equations (2) and (13)-(15). For a specified real Bloch wave vector $\mathbf{q}=\left\{q_{1}, q_{2}\right\}$ the problem is self-adjoint and the corresponding infinite sequence of eigenvalues $\lambda=\kappa^{2}$ of the negative Laplacian may be determined by a standard application of the Rayleigh-Ritz method (see, for example, Duff $\&$ Naylor, 1966, Chapter 6). The Rayleigh quotient for a given trial function $u$ is

$$
\mathcal{R}(u)=\frac{\int_{A}|\nabla u|^{2} \mathrm{~d} A}{\int_{A}|u|^{2} \mathrm{~d} A},
$$

where $A$ is the fluid domain within the horizontal cross section illustrated in figure 1. The Neumann condition (15) is a 'natural' boundary condition and need not be incorporated into the trial function $u$. However, it is essential that $u$ satisfies the Bloch condition (10). This is achieved by writing

$$
u=\sum_{m, n=-P}^{P} A_{m n} \mathrm{e}^{\mathrm{i}\left(\mathbf{q}+\mathbf{K}_{m n}\right) \cdot \mathbf{r}}
$$

where

$$
\mathbf{K}_{m n}=2 \pi\left(\frac{m}{L} \mathbf{i}+\frac{n}{W} \mathbf{j}\right)
$$

is a reciprocal lattice vector, as defined in equation (5), and $\mathbf{r}=x \mathbf{i}+y \mathbf{j}$ is the position vector of an arbitrary point in $A$. Approximations to the eigenvalues correspond to the local minima of $\mathcal{R}(u)$ 
with respect to variations in the coefficients $A_{m n}$. This leads to the generalised eigenvalue problem

$$
\sum_{m, n=-P}^{P}\left(\mathcal{E}_{k l m n}-\lambda \mathcal{H}_{k l m n}\right)=0, \quad k, l=-P, \ldots P
$$

for $\lambda$ where

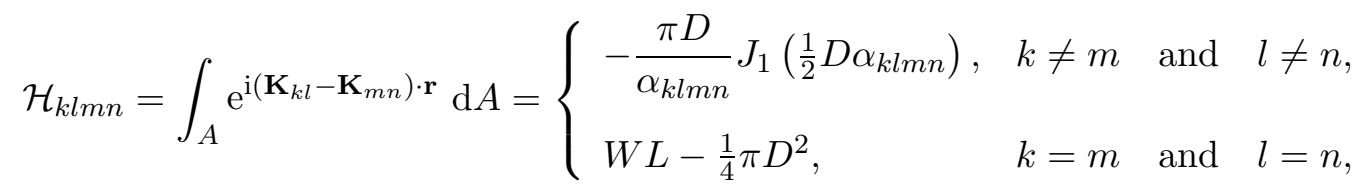

$$
\begin{aligned}
& \mathcal{E}_{k l m n}=\left[\left(q_{1}+2 \pi k / L\right)\left(q_{1}+2 \pi m / L\right)\right. \\
& \left.+\left(q_{2}+2 \pi l / W\right)\left(q_{2}+2 \pi n / W\right)\right] \int_{A} \mathrm{e}^{\mathrm{i}\left(\mathbf{K}_{k l}-\mathbf{K}_{m n}\right) \cdot \mathbf{r}} \mathrm{d} A \\
& =\left[\left(q_{1}+2 \pi k / L\right)\left(q_{1}+2 \pi m / L\right)+\left(q_{2}+2 \pi l / W\right)\left(q_{2}+2 \pi n / W\right)\right] \mathcal{H}_{k l m n}, \\
& \alpha_{k l m n}=2 \pi\left(\frac{(k-m)^{2}}{L^{2}}+\frac{(l-n)^{2}}{W^{2}}\right)^{1 / 2},
\end{aligned}
$$

and $J_{1}$ denotes the Bessel function of the first kind and order one.

The eigenvalue problem is solved using standard numerical techniques. The value of $P$ required to achieve a particular accuracy depends on the parameters $W / L$ and $D / L$. Most of the calculations in this paper are for $W / L=1$ and $D / L=0.5$; in this case $P=12$ is sufficient to obtain at least seven-figure accuracy for the twelve smallest eigenvalues. Smaller values of $D / L$ or values of $W / L$ significantly different from unity require larger values of $P$.

Although the above formulation uses $q_{1}$ and $q_{2}$ as input and then solves for $\kappa$, it is relatively straightforward to adapt the technique to use $q_{2}$ and $\kappa$ as input and then solve for $q_{1}$. Let $\left\{\kappa_{i}\left(q_{1}, q_{2}\right), i=1,2, \ldots\right\}$ denote the set of the square roots of the eigenvalues $\lambda$ corresponding to specified values of $q_{1}$ and $q_{2}$. Fix $q_{2}$ and for the chosen $\kappa$ define

$$
f\left(q_{1} ; \kappa, q_{2}\right)=\min _{i}\left|\kappa-\kappa_{i}\left(q_{1}, q_{2}\right)\right|
$$

Now determine the local minima of $f\left(q_{1} ; \kappa, q_{2}\right)$ as a function of $q_{1} \in[0, \pi / L]$. If a minimum of $f\left(q_{1} ; \kappa, q_{2}\right)$ is zero (within some tolerance), then the corresponding value of $q_{1}$ is a solution (there may be more than one such $\left.q_{1}\right)$. If the global minimum of $f\left(q_{1} ; \kappa, q_{2}\right)$ is not zero then, for the specified $q_{2}$, the chosen $\kappa$ is in a stopping band. 


\subsection{Results}

The case $q_{2}=0$ will be considered in some detail. This case can be interpreted by writing the potential in terms of parts that are symmetric and antisymmetric in $y$ so that

$$
\phi(x, y)=\phi_{S}(x, y)+\phi_{A}(x, y)
$$

where

$$
\phi_{S}(x, y)=\frac{1}{2}[\phi(x, y)+\phi(x,-y)]
$$

and

$$
\phi_{A}(x, y)=\frac{1}{2}[\phi(x, y)-\phi(x,-y)]
$$

and hence

$$
\frac{\partial \phi_{S}}{\partial y}(x, 0)=\phi_{A}(x, 0)=0 .
$$

From the Bloch conditions (14), it follows immediately that

$$
\begin{aligned}
\phi_{S}(x, W / 2) & =\frac{1}{2}\left[1+\mathrm{e}^{-\mathrm{i} q_{2} W}\right] \phi(x, W / 2) \\
\frac{\partial \phi_{S}}{\partial y}(x, W / 2) & =\frac{1}{2}\left[1-\mathrm{e}^{-\mathrm{i} q_{2} W}\right] \frac{\partial \phi}{\partial y}(x, W / 2) \\
\phi_{A}(x, W / 2) & =\frac{1}{2}\left[1-\mathrm{e}^{-\mathrm{i} q_{2} W}\right] \phi(x, W / 2) \\
\frac{\partial \phi_{A}}{\partial y}(x, W / 2) & =\frac{1}{2}\left[1+\mathrm{e}^{-\mathrm{i} q_{2} W}\right] \frac{\partial \phi}{\partial y}(x, W / 2)
\end{aligned}
$$

and so when $q_{2}=0$

$$
\frac{\partial \phi_{S}}{\partial y}(x, W / 2)=\frac{\partial \phi_{S}}{\partial y}(x,-W / 2)=\phi_{A}(x, W / 2)=\phi_{A}(x,-W / 2)=0 .
$$

Thus, solutions to the problem with $q_{2}=0$ correspond either to motions that are symmetric about $y=0$ and satisfy homogeneous Neumann conditions on $y= \pm W / 2$ (referred to here as type I modes), or to solutions that are antisymmetric about $y=0$ and satisfy homogeneous Dirichlet conditions on $y= \pm W / 2$ (type II modes). All solutions satisfy the Bloch conditions (13).

The type I and type II modes (as well as other modes) could be solved for separately by making appropriate choices of trial function with trigonometric functions. However, the determination of the form of the elements of the matrices in (30) is then a rather longer calculation. It is simpler to adopt the approach given above and identity the modes by computing the boundary values of the eigenvector and its normal derivative.

Calculations are now presented for various geometrical parameters in figures 2-4; the results are displayed in the reduced-zone scheme discussed in $\S 2$. Before presenting numerical results for 


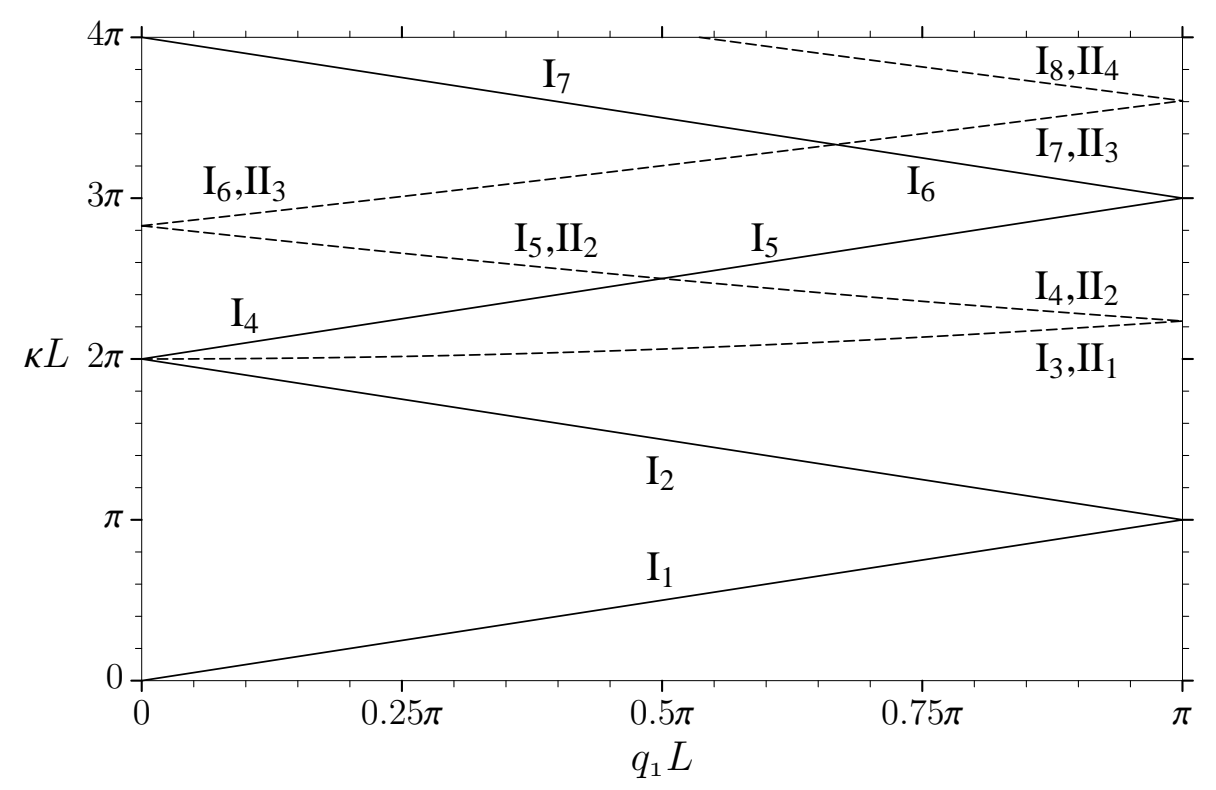

Figure 2: Eigenvalue $\kappa L$ vs. wave vector $q_{1} L ; W / L=1, D / L=0, q_{2} L=0$.

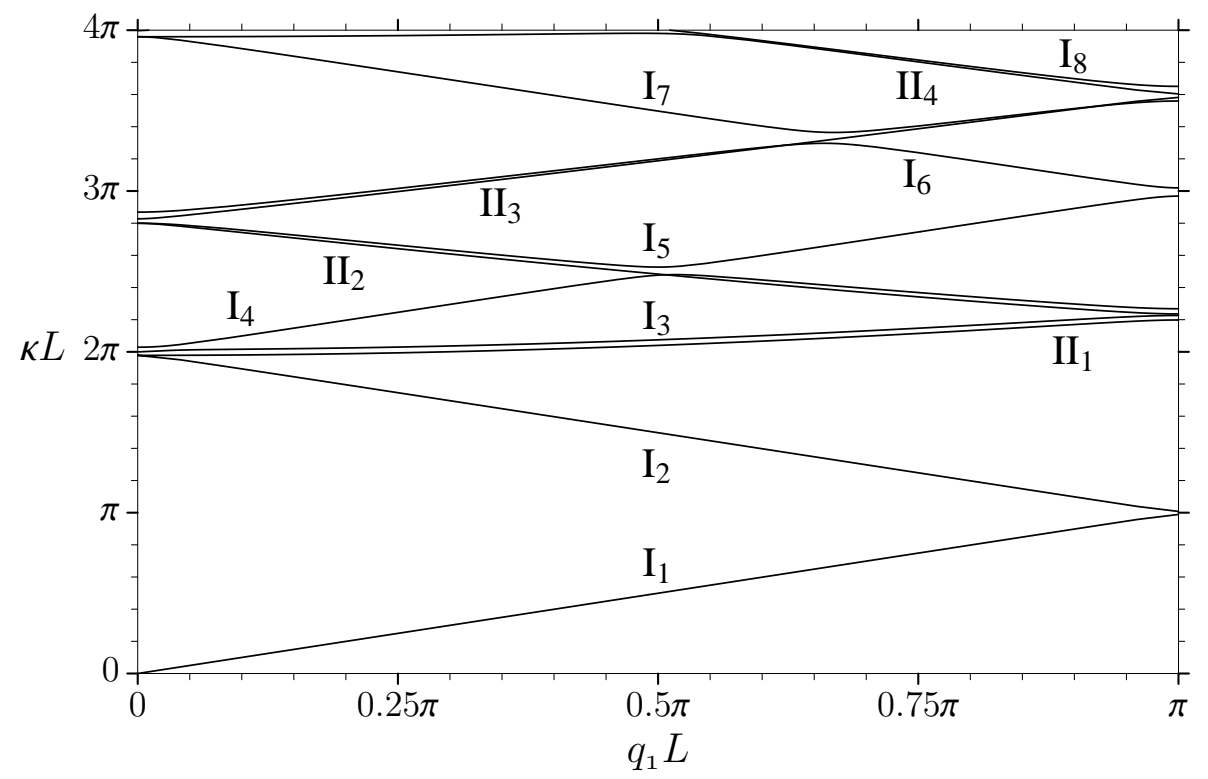

Figure 3: Eigenvalue $\kappa L v s$. wave vector $q_{1} L ; W / L=1, D / L=0.1, q_{2} L=0$.

the full problem, it is instructive to consider the problem when there is no cylinder present so that only the Bloch conditions need be satisfied. Attention will be restricted to modes with $\kappa W<4 \pi$; for any $q_{1}$, type I modes are

$$
\phi=\mathrm{e}^{\mathrm{i} q_{1} x}, \quad \kappa=q_{1},
$$




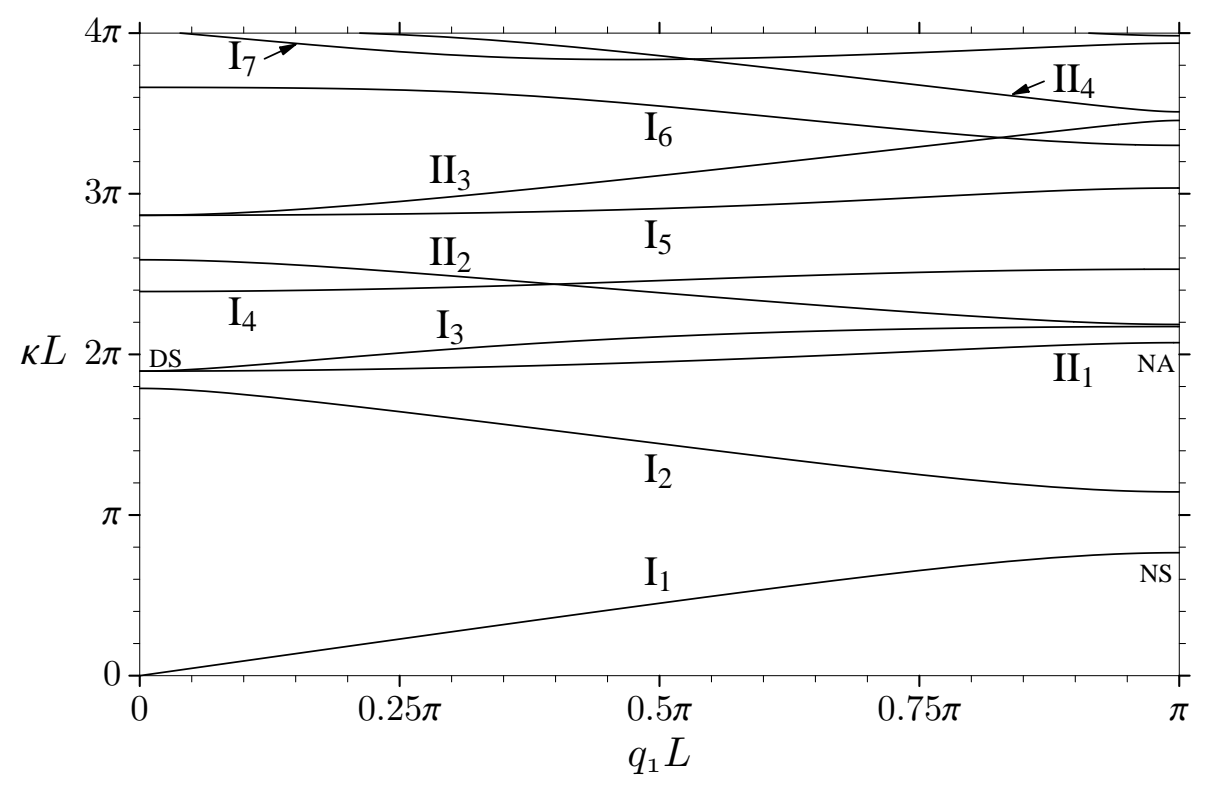

Figure 4: Eigenvalue $\kappa L$ vs. wave vector $q_{1} L ; W / L=1, D / L=0.5, q_{2} L=0$.

$$
\phi=\mathrm{e}^{\mathrm{i} q_{1} x} \cos \frac{2 \pi y}{W}, \quad \kappa=\left(q_{1}^{2}+\frac{4 \pi^{2}}{W^{2}}\right)^{1 / 2},
$$

and the only type II modes are

$$
\phi=\mathrm{e}^{\mathrm{i} q_{1} x} \sin \frac{2 \pi y}{W}, \quad \kappa=\left(q_{1}^{2}+\frac{4 \pi^{2}}{W^{2}}\right)^{1 / 2} .
$$

Clearly, the second type I mode and the type II mode share the same eigenvalue $\kappa$. Results for the case $W=L$ are displayed in figure 2 and each continuous curve linking $q_{1} L=0$ to $q_{1} L=\pi$, here called a branch, is labelled as either type I or type II. The branches of each mode type are indexed in strictly ascending order of $\kappa L$. Note that some branches of type I modes cross and the indexing has been chosen to reflect the behaviour observed when a cylinder is introduced.

For a cylinder of non zero but small radius, as in figure 3 , the pattern is superficially very similar. However, there are three significant differences. First of all, the type I and type II modes with identical eigenvalues in the absence of the cylinder have now split; identification of the branches was made by computing the corresponding eigenvector and verifying the boundary conditions. Secondly, the crossings of type I modes no longer exist. This has been verified carefully by increasing the accuracy of the computations in these regions. Finally, with non-zero $D / L$ there are so-called band gaps, although this is not immediately apparent from figure 3 as they are quite narrow. For example, for $0.99 \pi \lesssim \kappa L \lesssim 1.01 \pi$ there is no corresponding real value of $q_{1} L$. In other words, for $\kappa L$ in this range (and for $q_{2} L=0$ ) it is not possible for waves to propagate through the array without change of amplitude. 
For a larger radius, as in figure 4, the situation is more confused and it is not easy to identify the different modes by direct comparison with figure 2 . The labelling on the figure was arrived at by observing the changes as $D / L$ was increased through values not displayed here, and confirmed by computation of the eigenvector. The existence of band gaps is now clearly displayed, the first band gap has now widened to $0.77 \pi \lesssim \kappa L \lesssim 1.14 \pi$. The significance of these band gaps will be discussed later in $\S \S 6-7$.

For $q_{1} L$ equal to either zero or $\pi$, the solutions obtained may satisfy either homogeneous Dirichlet or homogeneous Neumann conditions on $x= \pm L / 2$. This can be seen explicitly by examining the symmetries of $\phi$ as in equations (39)-(42). In particular, $q_{1} L=0$ can correspond to either the symmetric Neumann modes or the antisymmetric Dirichlet modes while $q_{1} L=\pi$ can correspond to either the antisymmetric Neumann or the symmetric Dirichlet modes. Similar comments apply to $q_{2} W$ and the cell boundaries at $y= \pm W / 2$. Hence, for $q_{1} L$ and $q_{2} W$ both equal to one of zero or $\pi$, the solutions obtained may correspond to standing waves satisfying the same homogeneous boundary condition on opposite sides of the cell. To obtain other standing-wave solutions satisfying, for example, a homogeneous Neumann condition on $x=-L / 2$ and a homogeneous Dirichlet condition on $x=L / 2$ the underlying periodicity of the solutions must be increased to accommodate a cell of twice the size.

\subsection{Connection with trapped modes}

The variational method is now used to investigate how so-called 'trapped modes' are recovered as one of the cell dimensions, $W$ say, is allowed to increase indefinitely. A trapped mode is a free oscillation of finite energy within an unbounded fluid. Callan, Linton \& Evans (1991) proved that such trapped modes may exist within a rigid, parallel-walled channel of infinite length that has a rigid cylinder symmetrically placed about the centre line. Subsequently, trapped modes have been found to exist when homogeneous Dirichlet conditions are applied on the channel walls (this is non-physical within the context of water waves in a channel, but the problem can be interpreted in terms of wave interaction with arrays of cylinders; see Maniar \& Newman, 1997).

Evans \& Porter (1999) give a review of the present state of knowledge regarding trapped modes supported by a rigid cylinder in a channel, and the following summarises some of these results using their notation for mode identification. Modes satisfying Neumann (Dirichlet) conditions on the channel walls, here $x= \pm L / 2$, are denoted by $\mathrm{N}(\mathrm{D})$ and modes that are symmetric (antisymmetric) about $y=0$ are denoted by $\mathrm{S}(\mathrm{A})$. (In view of equations (39)-(42), for the Bloch problem this classification is sufficient to identify all boundary conditions applied on the cell perimeter and all symmetries about $x, y=0$.) For some $\kappa L \in(0, \pi)$ an NS trapped mode exists for any $D / L$, 


\begin{tabular}{ccccc}
$W / L$ & $\kappa L(0.5, \mathrm{NS})$ & $\kappa L(0.9, \mathrm{NS})$ & $\kappa L(0.9, \mathrm{NA})$ & $\kappa L(0.5, \mathrm{DS})$ \\
\hline 1 & $0.765 \pi$ & $0.526 \pi$ & $2.053 \pi$ & $1.896 \pi$ \\
2 & $0.860 \pi$ & $0.818 \pi$ & $1.149 \pi$ & $1.942 \pi$ \\
3 & $0.879 \pi$ & $0.851 \pi$ & $1.031 \pi$ & $1.951 \pi$ \\
4 & $0.884 \pi$ & $0.859 \pi$ & $1.002 \pi$ & $1.954 \pi$ \\
5 & $0.885 \pi$ & $0.860 \pi$ & $0.992 \pi$ & $1.955 \pi$ \\
$\infty$ & $0.886 \pi$ & $0.861 \pi$ & $0.99 \pi$ & $1.956 \pi$
\end{tabular}

Table 1: Approach of standing-wave frequencies to trapped-mode frequencies as the cell aspect ratio $W / L \rightarrow \infty$. The number in parentheses is the cylinder diameter $D / L$ and the letters identify the mode (see the text).

but an NA mode exists only for $D / L \gtrsim 0.81$. For some $\kappa L \in(0,2 \pi)$ a DS mode exists for all $D / L \lesssim 0.68$, but no corresponding DA mode has been found. All of these modes are antisymmetric about the centre-line of the channel $x=0$ and are below the first appropriate cut-off frequency for antisymmetric propagating modes. The cut-off for Neumann modes is $\kappa L=\pi$ and for Dirichlet modes it is $\kappa L=2 \pi$.

The notation described above has been used to identify some of the standing-wave modes in figure 4. For $q_{1} L=0$ there is a double eigenvalue at $\kappa L \simeq 1.896 \pi$ and the DS mode is the limit of the $\mathrm{I}_{3}$ curve as $q_{1} L \rightarrow 0$. Data showing the approach to trapped modes as $W / L$ increases is given in table 1. The trapped mode frequencies $(W / L \rightarrow \infty)$ are taken from Callan et al. (1991) for the NS modes, from Maniar \& Newman (1997) for the DS mode, and from Evans \& Porter (1999) for the NA mode. The latter value was estimated from graphical results and so is given to only two significant figures.

Consider first the modes corresponding to $q_{1} L=\pi$. For $D / L=0.5$, as $W / L \rightarrow \infty$ the NS mode marked in figure 4 remains the only mode below $\kappa L=\pi$. All other modes remain above $\kappa L=\pi$, and will tend asymptotically to a cut-off at $\kappa L=n \pi$ for some positive integer $n$ (under the assumption that there are no embedded trapped modes for this geometry). For this NS mode, the convergence of $\kappa L$ to the trapped mode value as $W / L$ increases is shown in table 1 . For $D / L=0.5$, the marked NA mode does not become a trapped mode. However, for $D / L=0.9$, as $W / L \rightarrow \infty$ the lowest NS and NA modes both asymptote to trapped modes below the cut-off at $\kappa L=\pi$ as shown in table 1. Again, all other modes appear to remain above the cut-off as $W / L$ is increased.

The eigenvalues are clearly very close to the trapped-mode frequencies for $W / L=5$. The change in the approximate eigenfunction $u$ (calculated from equation 28) as $W / L$ increases is illustrated in figure 5 for an NS mode showing that, as $W / L \rightarrow \infty, u$ becomes increasingly concentrated around 


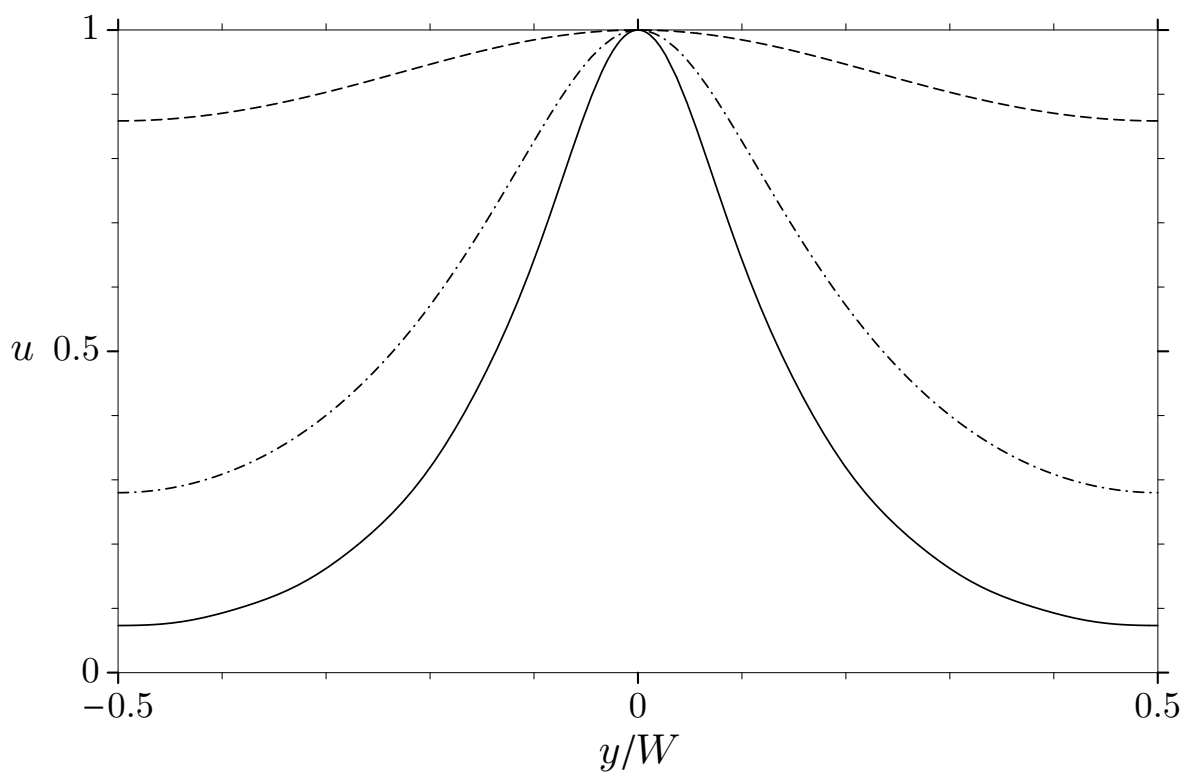

Figure 5: Approximate eigenfunction $u$ (NS mode) vs. $y / W$ on $x=L / 2$ for $W / L=1$ $(---), W / L=3(-\cdot-\cdot-)$, and $W / L=5(\longrightarrow) ; D / L=0.5, q_{2} L=0$.

$y=0$ and seems to approach zero at $y / W= \pm 1 / 2$, which is the expected behaviour if the limit is to be a trapped mode.

The final mode marked in figure 4 is the DS mode corresponding to $q_{1} L=0$. The antisymmetric propagating wave cut-off is at $\kappa L=2 \pi$ when Dirichlet conditions are applied on the channel walls and the trapped mode value of $\kappa L$ is now a little below $2 \pi$ (see table 1 ). As $W / L$ is increased, more and more modes move below $\kappa L=2 \pi$. Calculations suggest that, with the exception of the DS mode, these modes all satisfy Neumann conditions on $x= \pm L / 2$ and are symmetric about $x=0$; in an infinite channel there is no positive cut-off frequency for such modes and so in general the potential will not decay to zero as $|y| \rightarrow \infty$. Thus, it seems that the single DS mode is the only mode that can asymptote to a trapped mode for $q_{1} L=0$ and $\kappa L<2 \pi$.

Note that standing waves that asymptote to trapped modes can be found irrespective of whether a Neumann or Dirichlet boundary condition is applied on $y= \pm W / 2$ (that is whether $q_{2} W=0$ or $q_{2} W=\pi$ respectively). This topic and the nature of the approach to a trapped mode will be investigated further in another paper.

\subsection{Connection with Rayleigh-Bloch waves}

A generalisation of a trapped mode is the so-called 'Rayleigh-Bloch' wave. Such waves may propagate along an infinite row of equally-spaced, rigid, vertical cylinders with decay of the fluid motion to zero in the direction normal to the row. Evans \& Porter (1999) give a review of the present 


\begin{tabular}{c|ccccccccc}
$q_{1} L$ & $\frac{1}{5} \pi$ & $\frac{1}{4} \pi$ & $\frac{1}{3} \pi$ & $\frac{2}{5} \pi$ & $\frac{1}{2} \pi$ & $\frac{3}{5} \pi$ & $\frac{2}{3} \pi$ & $\frac{3}{4} \pi$ & $\frac{4}{5} \pi$ \\
\hline$\kappa L(\mathrm{BB})$ & $0.197 \pi$ & $0.246 \pi$ & $0.328 \pi$ & $0.394 \pi$ & $0.491 \pi$ & $0.588 \pi$ & $0.651 \pi$ & $0.728 \pi$ & $0.773 \pi$ \\
$\kappa L(\mathrm{RB})$ & $0.200 \pi$ & $0.250 \pi$ & $0.332 \pi$ & $0.398 \pi$ & $0.497 \pi$ & $0.594 \pi$ & $0.657 \pi$ & $0.733 \pi$ & $0.777 \pi$
\end{tabular}

Table 2: Comparison between the present calculations (BB) and Rayleigh-Bloch wave frequencies $(\mathrm{RB}) ; W / L=5, D / L=0.5, q_{2} L=0$.
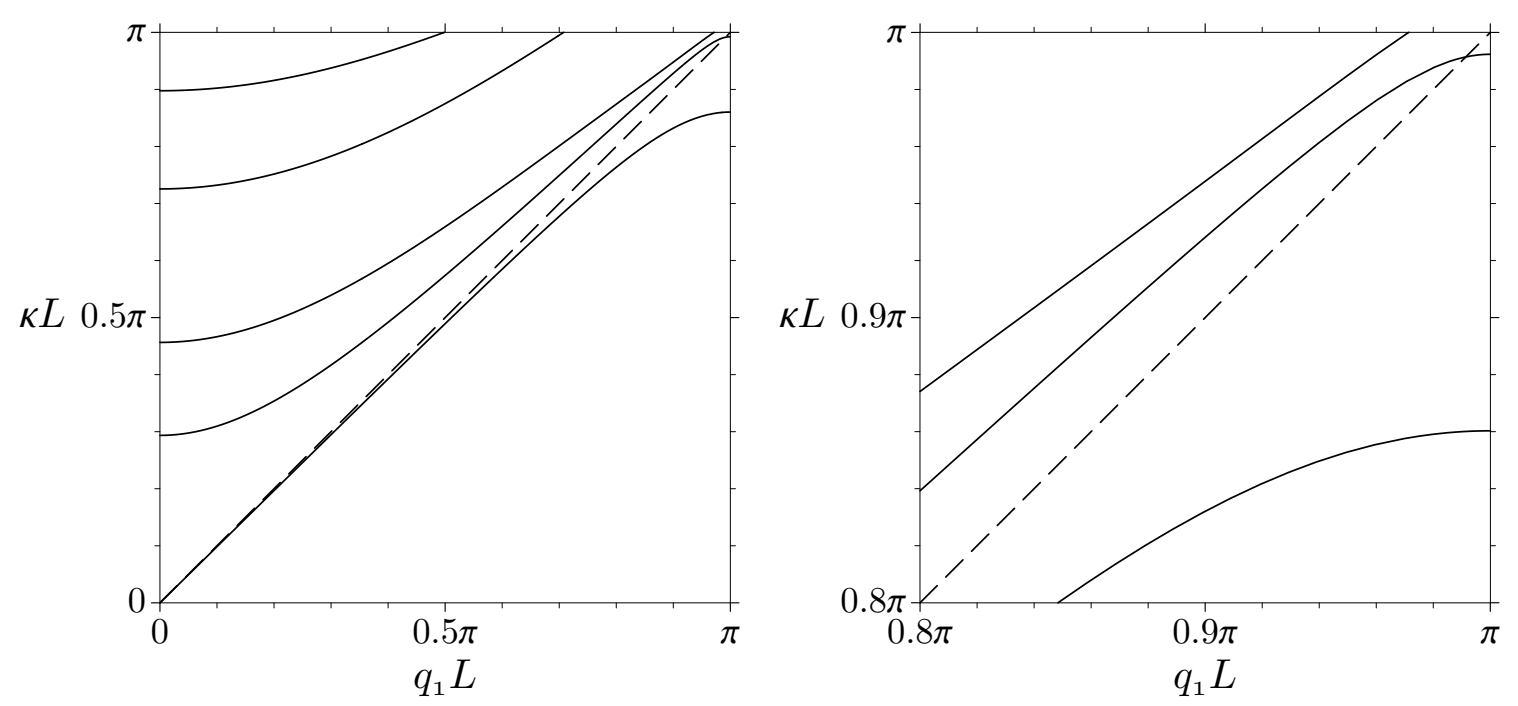

Figure 6: Eigenvalue $\kappa L$ vs. wave vector $q_{1} L ; W / L=5, D / L=0.9, q_{2} L=0$. The right-hand figure is an enlargement of part of the left-hand figure. The dashed line is the cut-off $\kappa L=q_{1} L$.

state of knowledge concerning Rayleigh-Bloch waves of this type and they report two types of Rayleigh-Bloch wave that, for a fixed $q_{1} L \leq \pi$, exist for discrete $\kappa L \in\left(0, q_{1} L\right)$. These are waves symmetric about $x=0$ that exist for all non-dimensional cylinder diameters $D / L \in(0,1]$, and waves antisymmetric about $x=0$ that exist only when $0.81 \lesssim D / L \leq 1$. As for trapped modes, the numerical evidence suggests that the frequencies of Rayleigh-Bloch waves are recovered in the present problem by taking the limit $W / L \rightarrow \infty$. Comparison between the present calculations and those for symmetric Rayleigh-Bloch waves made by Porter \& Evans (1999, table 2) is made in table 2 .

Another example is illustrated in figure 6. For $D / L=0.9$, all but two of the frequency curves remain above the cut-off at $\kappa L=q_{1} L$. The complete curve below the cut-off corresponds to symmetric Rayleigh-Bloch waves and the partial curve to antisymmetric Rayleigh-Bloch waves. The latter curve is close to the appropriate curve in figure 10(b) of Evans \& Porter (1999). Calculations 
for $D / L=0.5$ are reported by Evans \& Porter (1999) and McIver, Linton \& McIver (1998) and their graphical results may be compared with the $\mathrm{I}_{1}$ curve in the present figure 4 .

The limiting values as $q_{1} L \rightarrow \pi$ of the Rayleigh-Bloch wavenumbers $\kappa L$ corresponding to the two lowest curves in figure 6 are just the NS and NA trapped modes for $D / L=0.9$ given in table 1 . All known Rayleigh-Bloch waves become trapped waves in this limit. The DS mode discussed in $\S 4.3$ is not the limit of any known Rayleigh-Bloch wave and it may be that there is a hitherto undiscovered Rayleigh-Bloch wave for which the DS mode is the limit.

\section{$5 \quad$ Numerical calculations within the stopping bands}

The method of $\S 4$ is suitable only for calculations within passing bands. In this section an outline is given of two standard numerical methods that can be used for calculations within stopping bands.

\subsection{Point-matching formulation}

For specified $\kappa$ and $q_{2}$, the problem defined by equations (2) and (13)-(15) may be formulated as an eigenvalue problem for $q_{1}$ as follows. The series

$$
\phi=A_{0} C_{0}(\kappa r)+\sum_{n=1}^{N} C_{n}(\kappa r)\left(A_{n} \cos n \theta+B_{n} \sin n \theta\right),
$$

where

$$
C_{n}(\kappa r)=J_{n}(\kappa r) Y_{n}^{\prime}(\kappa D / 2)-Y_{n}(\kappa r) J_{n}^{\prime}(\kappa D / 2),
$$

satisfies both the field equation (2) and the cylinder boundary condition (15). The remaining Bloch conditions $(13)-(14)$ are satisfied pointwise. Let $(r, \theta)=\left(r_{i}, \theta_{i}\right)$ be the polar coordinates of $P+Q$ points on two sides of the primitive cell $\{|x| \leq L / 2,|y| \leq W / 2\}$. The first $P$ points are on $\{x=L / 2,|y| \leq W / 2\}$ and, in order to apply the Bloch conditions, complementary points $(r, \theta)=\left(r_{i}, \pi-\theta_{i}\right)$ are required on $\{x=-L / 2,|y| \leq W / 2\}$. The remaining $Q$ points are on $\{y=W / 2,|x| \leq L / 2\}$ with complementary points $(r, \theta)=\left(r_{i},-\theta_{i}\right)$ on $\{y=-W / 2,|x| \leq L / 2\}$.

Application of the Bloch conditions at these points leads to a system of equations for the unknown coefficients $\left\{A_{0}, A_{1}, \ldots, A_{N}, B_{1}, \ldots, B_{N}\right\}=\mathbf{x}^{T}$, say. If the conditions are satisfied in the least-squares sense then a necessary condition for a minimum of the sum of squares of the residuals with respect to $\mathbf{x}$ has the form

$$
A \mathrm{x}=\mathbf{0}
$$

where $A$ is a $(2 N+1) \times(2 N+1)$ matrix. There is a non-trivial solution for $\mathbf{x} \operatorname{provided} \operatorname{det} A=0$. Rather than seek a zero of $\operatorname{det} A$, values of $\kappa L$ and $q_{2} W$ are specified and $|\operatorname{det} A| \operatorname{minimised}$ as a 
function of $q_{1} L$. This formulation works very well for $\kappa W \lesssim 2 \pi$. Within this range, an accuracy for $q_{1} L$ of a three digits or more is usually obtained for $N=16$ and $P=Q=4 N$.

The system of equations resulting from application of the Bloch conditions may also be rewritten as a generalised eigenvalue problem for the eigenvalue $\mathrm{e}^{\mathrm{i} q_{1} L}$ which can be solved using the QZ algorithm (Golub \& Van Loan, 1983). A reduction to a standard eigenvalue problem is not possible in general as the matrices involved become nearly singular as $P$ and $Q$ are increased. The method works well for $\kappa L \lesssim 4 \pi$, but for $\kappa L \gtrsim 4 \pi$ large values of $N, P$ and $Q$ are required to obtain reasonable accuracy and the QZ algorithm becomes unreliable and may converge to erroneous values if insufficient care is taken. With this in mind, a more robust, but more expensive, method of computation is described in the next section.

\subsection{Boundary-integral formulation}

For given $\kappa$ and $q_{2}$, the problem defined by equations (2) and (13)-(15) may be formulated as an eigenvalue problem for $q_{1}$ using an application of Green's theorem to the potential $\phi$ and a suitable Green's function $G$. The Green's function is chosen to satisfy the cylinder-surface boundary condition (15) and is

$$
G(x, y ; \xi, \eta)=H_{0}(\kappa R)-\sum_{n=0}^{\infty} \epsilon_{n} \frac{J_{n}^{\prime}(\kappa D / 2)}{H_{n}^{\prime}(\kappa D / 2)} H_{n}(\kappa \rho) H_{n}(\kappa r) \cos n(\theta-\psi) .
$$

Here, $(\rho, \psi)$ and $(\xi, \eta)$ are respectively the polar and Cartesian coordinates of the source point $P$, $(r, \theta)$ and $(x, y)$ are the corresponding coordinates of the field point $Q, R$ is the distance between $P$ and $Q$ and satisfies

$$
R^{2}=(x-\xi)^{2}+(y-\eta)^{2}=r^{2}+\rho^{2}-2 r \rho \cos (\theta-\psi)
$$

$J_{n}$ denotes the Bessel function of the first kind and order $n, H_{n}$ denotes the Hankel function of the first kind and order $n, \epsilon_{0}=1$, and $\epsilon_{n}=2$ for $n \geq 1$. That $G$ satisfies the boundary condition (15) may be verified using Graf's addition theorem (Abramowitz \& Stegun, 1964, equation 9.1.79).

With the above choice of Green's function, Green's theorem yields

$$
\phi(P)=\frac{1}{2 i} \int_{S}\left(\phi(Q) \frac{\partial G}{\partial n_{Q}}(P ; Q)-G(P ; Q) \frac{\partial \phi}{\partial n_{Q}}(Q)\right) \mathrm{d} s_{Q}
$$

where the integration is taken over the boundary $S$ of the rectangular region $\{|x| \leq L / 2,|y| \leq W / 2\}$, and the subscript $Q$ on the outward normal coordinate $n$ and the tangential coordinate $s$ is used to denote derivatives with respect to the field variables. Substitution of the representation (52) into the conditions on $\phi$ in equations (13)-(14), and application of all of (13)-(14) under the integral 
sign, gives a set of four coupled integral equations for the four unknown functions

$$
\begin{array}{clrl}
f(y) \equiv \phi(L / 2, y), & u(y) \equiv \frac{\partial \phi}{\partial x}(L / 2, y), & |y| \leq W / 2, \\
g(x) \equiv \phi(x, W / 2), & v(x) \equiv \frac{\partial \phi}{\partial y}(x, W / 2), & |x| \leq L / 2 .
\end{array}
$$

Divide the interval $|y| \leq W / 2$ in to $M$ elements of equal length and the interval $|x| \leq L / 2$ in to $N$ elements of equal length. The functions $f(y), u(y), g(x)$ and $v(x)$ are assumed to be constant over the appropriate individual elements which allows the simultaneous integral equations to be reduced in a standard way to a matrix system for the unknown function values that has the form

$$
A\left(\lambda_{2}, \kappa L\right) \mathbf{x}=\lambda_{1} B\left(\lambda_{2}, \kappa L\right) \mathbf{x}
$$

where $\lambda_{1}=\mathrm{e}^{\mathrm{i} q_{1} L}, \lambda_{2}=\mathrm{e}^{\mathrm{i} q_{2} W}, A$ and $B$ are matrices of order $2(N+M) \times 2(N+M)$, and $\mathbf{x}$ is the vector of unknown function values. Equation (55) is a generalised eigenvalue problem for the eigenvalue $\lambda_{1}$ which can be solved by the QZ algorithm (Golub \& Van Loan, 1983). Significant reduction in computational time can be obtained by reducing the size of the system by elimination of $\mathbf{f}$ and $\mathbf{u}$ from (55). Note that a reduction to a standard eigenvalue problem is not possible as the matrices involved become nearly singular as $M$ and $N$ increase. Computations for a square cell suggest that $q_{1} L$ may be computed to about three-figure accuracy for $\kappa L \lesssim 4 \pi$ by taking $M=N=16$. The boundary-integral method is recommended for calculations of $T_{\mathrm{B}}$ in the stopping bands for $\kappa L \gtrsim 2 \pi$.

\subsection{Results}

Typical results for the Bloch transmission coefficient $T_{\mathrm{B}}=\mathrm{e}^{\mathrm{i} q_{1} L}$ (see $\S 3$ ) are illustrated in figure 7 . The ranges $0<\kappa L \lesssim 0.77 \pi, 1.14 \pi \lesssim \kappa L \lesssim 1.79 \pi$ and $1.90 \pi \lesssim \kappa L<2 \pi$, are passing bands where $\left|T_{\mathrm{B}}\right|=1$ so that the waves propagate through the array with constant amplitude. The complementary ranges $0.77 \pi \lesssim \kappa L \lesssim 1.14 \pi$ and $1.79 \pi \lesssim \kappa L \lesssim 1.90 \pi$ are stopping bands in which $\left|T_{\mathrm{B}}\right|<1$ so that the waves decay in amplitude as they propagate. The passing bands were first identified using the method of $\S 4.1$ and then $T_{\mathrm{B}}$ was computed in the stopping bands using the above least-squares formulation.

Figure 8 shows how the real and imaginary parts of $q_{1} L$ vary with $\kappa L$ for the same geometry as used in figure 7. As $\kappa L$ is increased in the first passing band, $\operatorname{Im}\left\{q_{1} L\right\}=0$ and the $\mathrm{I}_{1}$ mode is followed up to the first stopping band. Within the stopping band the phase $\operatorname{Re}\left\{q_{1} L\right\}$ remains constant while $\operatorname{Im}\left\{q_{1} L\right\}$ varies. The $\mathrm{I}_{2}$ mode is then followed until the second stopping bound where $\operatorname{Re}\left\{q_{1} L\right\}$ again remains constant. After the second stopping band there are two modes; the $\mathrm{II}_{1}$ mode 


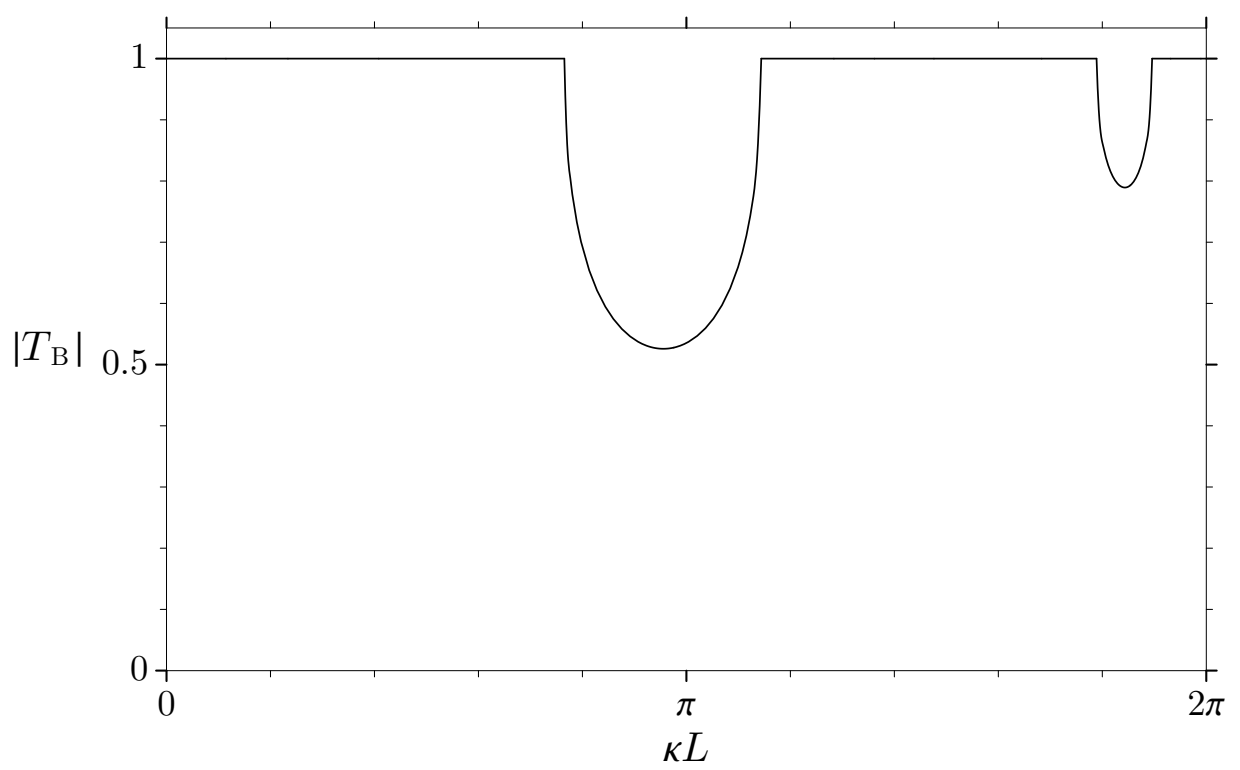

Figure 7: Bloch transmission coefficient $\left|T_{\mathrm{B}}\right|$ vs. wavenumber $\kappa L ; W / L=1, D / L=0.5, q_{2} L=0$.

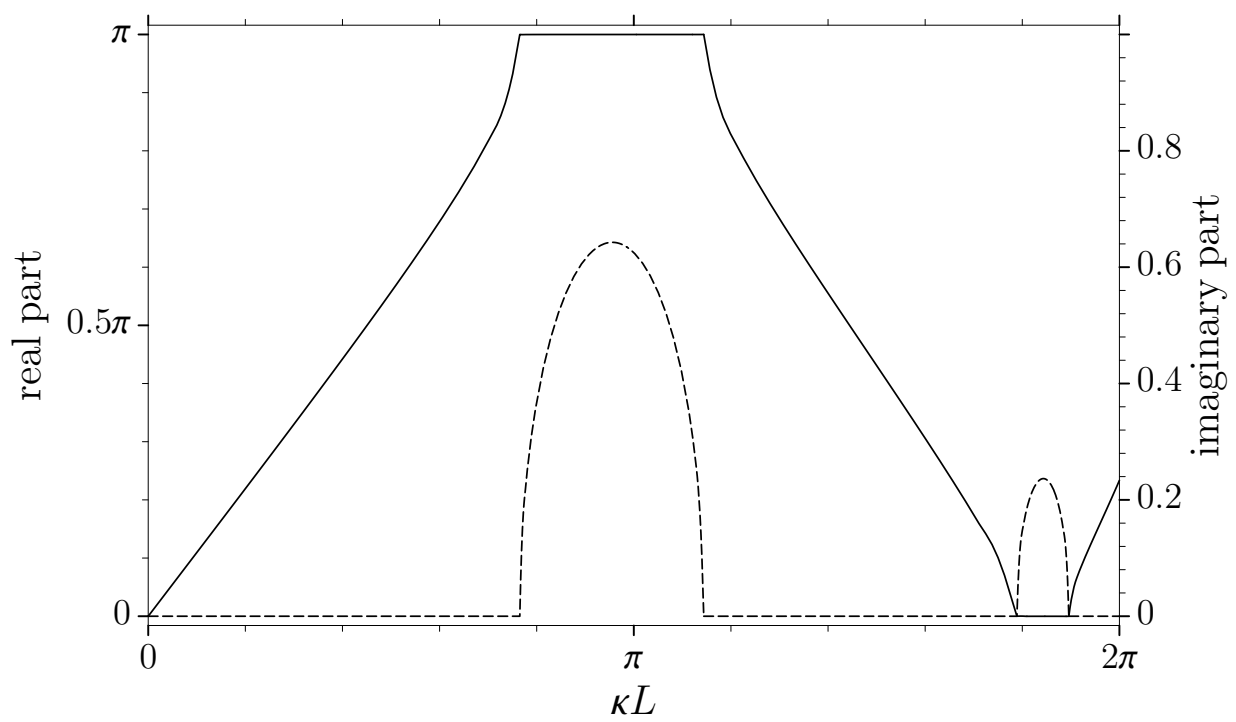

Figure 8: Real (- $)$ and imaginary $(----)$ parts of $q_{1} L$ vs. wavenumber $\kappa L$; $W / L=1, D / L=0.5, q_{2} L=0$.

is followed in figure 8. The behaviour obtained here from numerical calculations confirms exactly that observed in the approximate solution of $\S 3$ that is based upon a wide-spacing approximation. 


\section{Unidirectional wave propagation through an array}

The behaviour described in the preceding sections for a doubly infinite array is related to the reflection and transmission properties of an array that has finite length in the $x$ direction. This is illustrated here for normal incidence, $q_{2}=0$, and $\kappa W<2 \pi$ using the wide-spacing formalism of Evans (1990). Consider $N$ infinitely long rows of cylinders situated at $x=L_{m}, m=1,2, \ldots N$. Each row may be thought of as a single cylinder with axis on $y=0$ between channel walls at $y= \pm W / 2$. Divide the fluid domain into $N+1$ regions as follows:

$$
\begin{array}{ll}
\text { Region } 1: & -\infty<x<L_{1}, \\
\text { Region } \mathrm{m}: & L_{m-1}<x<L_{m}, m=2,3, \ldots, N, \\
\text { Region } \mathrm{N}+1: & L_{N}<x<\infty .
\end{array}
$$

Here $L_{m+1}-L_{m}=L, m=1,2, \ldots, N-1$. It will be assumed that $\kappa L>>1$. Suppose that an incident plane wave propagates in the direction of $x$ increasing and this is reflected and transmitted by the complete grating. Under the wide-spacing approximation, at sufficiently large distances from the cylinders, in region $m$ the solution may be written as

$$
\phi=A_{m} \mathrm{e}^{\mathrm{i} \kappa x}+B_{m} \mathrm{e}^{-\mathrm{i} \kappa x} .
$$

In region 1 this will correspond to the incident and reflected wave for the complete grating and in region $N+1$ to the transmitted wave. The solutions in region $m$ and region $m+1$ may be related to the reflection and transmission coefficients for a single row, $R$ and $T$ respectively, in the same way as described before equations (20). This leads to the equations

$$
\begin{aligned}
& A_{1}=1, \quad B_{1}=R_{N}, \quad A_{N+1}=T_{N}, \quad B_{N+1}=0, \\
& \left.\begin{array}{l}
A_{m+1} \mathrm{e}^{\mathrm{i} \kappa L_{m}}=T A_{m} \mathrm{e}^{\mathrm{i} \kappa L_{m}}+R B_{m+1} \mathrm{e}^{-\mathrm{i} \kappa L_{m}} \\
B_{m} \mathrm{e}^{-\mathrm{i} \kappa L_{m}}=T B_{m+1} \mathrm{e}^{-\mathrm{i} \kappa L_{m}}+R A_{m} \mathrm{e}^{\mathrm{i} \kappa L_{m}}
\end{array}\right\}, \quad m=1,2, \ldots, N,
\end{aligned}
$$

where $R_{N}$ and $T_{N}$ are the reflection and transmission coefficients for the complete array of $N$ rows. The steps described by Evans (1990) allow this system to be rewritten in the form

$$
\left(\begin{array}{c}
T_{N} \mathrm{e}^{\mathrm{i}\left(\kappa L_{N}+\kappa L\right)} \\
0
\end{array}\right)=S^{N}\left(\begin{array}{c}
\mathrm{e}^{\mathrm{i} \kappa L_{1}} \\
R_{N} \mathrm{e}^{-\mathrm{i} \kappa L_{1}}
\end{array}\right)
$$

where the matrix

$$
S=\left(\begin{array}{cc}
\left(T-R^{2} / T\right) \mathrm{e}^{\mathrm{i} \kappa L} & R \mathrm{e}^{\mathrm{i} \kappa L} / T \\
-R \mathrm{e}^{-\mathrm{i} \kappa L} / T & \mathrm{e}^{-\mathrm{i} \kappa L} / T
\end{array}\right)
$$


the system is easily solved to determine $R_{N}$ and $T_{N}$. Let

$$
S^{N} \equiv\left(\begin{array}{ll}
s_{11} & s_{12} \\
s_{21} & s_{22}
\end{array}\right)
$$

then, in particular,

$$
T_{N}=\mathrm{e}^{\mathrm{i} \kappa\left\{L_{1}-L_{N}-L\right\}}\left(s_{11}-\frac{s_{12} s_{21}}{s_{22}}\right) .
$$

For purely numerical purposes it is straightforward to evaluate $S^{N}$ directly. However, Evans (1990) gives an explicit formula for $S^{N}$ which yields further insight into the problem. From Evans' result it is easy to deduce that

$$
s_{11}-\frac{s_{12} s_{21}}{s_{22}}=\frac{\sinh \beta}{\sinh (\beta-N \alpha)}
$$

where

$$
\begin{aligned}
& \cosh \alpha=\frac{\mathrm{e}^{\mathrm{i} \kappa L}}{2 T}\left(T^{2}-R^{2}+\mathrm{e}^{-2 \mathrm{i} \kappa L}\right)=\frac{\cos (\delta+\kappa L)}{|T|}, \\
& \cosh \beta=\frac{\mathrm{e}^{\mathrm{i} \kappa L}}{2 R}\left(T^{2}-R^{2}-\mathrm{e}^{-2 \mathrm{i} \kappa L}\right)=\frac{\sin (\delta+\kappa L)}{|R|}
\end{aligned}
$$

and $\delta$ is the phase of the transmission coefficient introduced in equation (22). From equation (26) it may be seen that $\alpha$ is directly related to the Bloch wavenumber $q_{1}$ in the corresponding periodic-array problem.

At wave frequencies for which $|T|<|\cos (\delta+\kappa L)|, \alpha$ has a non-zero real part and $\beta$ is pure imaginary. Let $\beta=\mathrm{i} b$ so that

$$
\left|T_{N}\right|^{2}=\frac{\sin ^{2} b}{\sin ^{2} b+\sinh ^{2} N \alpha}
$$

and it is readily apparent that $\left|T_{N}\right| \rightarrow 0$ as $N \rightarrow \infty$. The frequencies at which this behaviour occurs correspond precisely to the stopping bands in the infinite-array problem. At wave frequencies for which $|T|>|\cos (\delta+\kappa L)|, \alpha=\mathrm{i} q_{1} L$ is pure imaginary and $\cosh \beta$ and $\sinh \beta$ are both real. Now

$$
\left|T_{N}\right|^{2}=\frac{\sinh ^{2} \beta}{\sinh ^{2} \beta+\sin ^{2} N q_{1} L} .
$$

and $\left|T_{N}\right|$ is oscillatory. The frequencies at which this behaviour occurs correspond precisely to the passing bands in the infinite-array problem.

The above behaviour of $T_{N}$ is confirmed in figure 9 using results for a grating with $N=10$ rows. The required reflection and transmission coefficients for a single cylinder were calculated by the method of Linton \& Evans (1993). Comparison is made with $\left|T_{\mathrm{B}}\right|^{10}$ which is the transmission coefficient for propagation through a distance $10 L$ in the doubly infinite array. The oscillatory behaviour in $\left|T_{10}\right|$ is due to end effects for the finite number of rows. Clearly, the Bloch transmission 


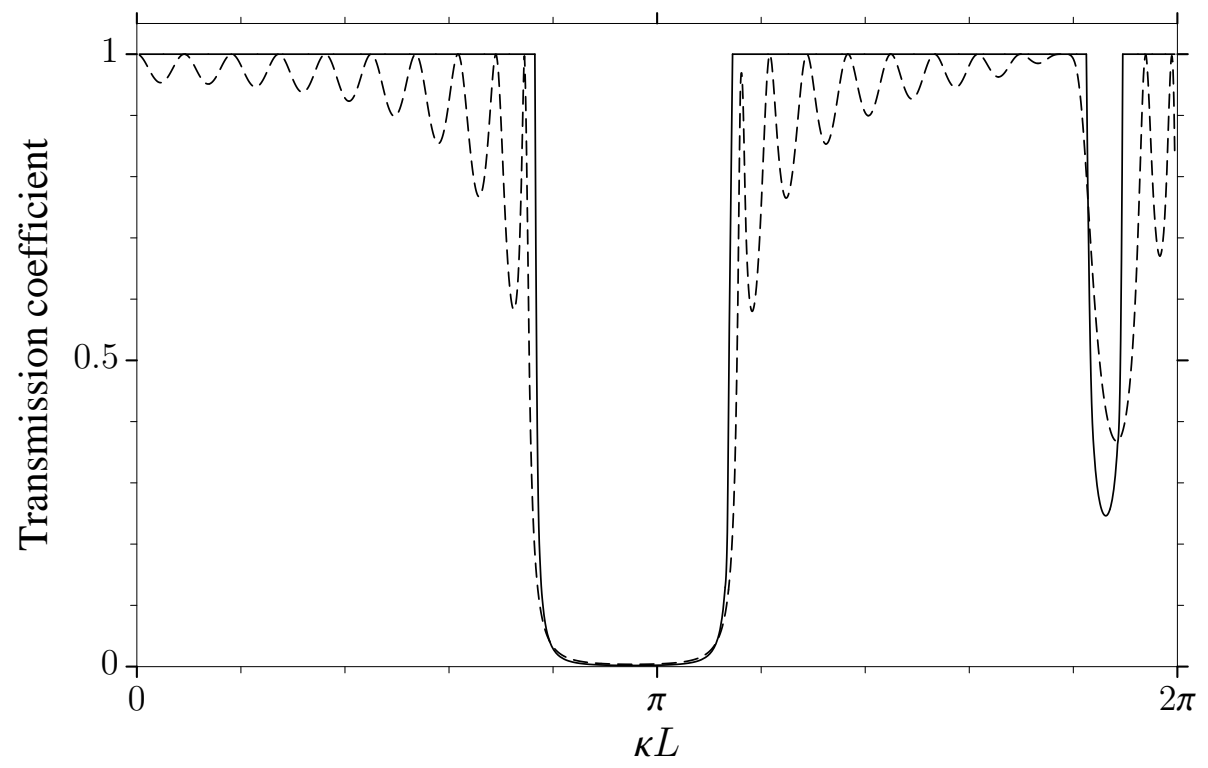

Figure 9: Transmission coefficient $\left|T_{10}\right|$ for ten rows of cylinders (dashed line) and Bloch transmission coefficient $\left|T_{\mathrm{B}}\right|^{10}$ (solid line) vs. wavenumber $\kappa L ; W / L=1$, $D / L=0.5$.

coefficient may be used to predict the properties of a large, but finite, number of rows of cylinders. Similar numerical comparisons of the Bloch transmission coefficient with the transmission by finite arrays have been made by Heckl \& Mulholland (1995) in the context of acoustic transmission in tube bundles.

The approximate positions of the troughs in transmission (and hence peaks in reflection) can be explained through the phenomenon of Bragg scattering that is well-known in x-ray diffraction by a crystal (Ashcroft \& Mermin, 1976, p.96). For strong overall reflection to occur the waves reflected from different rows of a grating must interfere constructively and, for the normal incidence investigated here, this occurs when $\kappa L=n \pi$, for integer $n$, which is clearly consistent with the results of figure 9 .

\section{Multi-directional wave propagation through an array}

The previous section dealt with normal incidence on a grating and with frequencies where the reflected and transmitted waves are also normal to the grating. This section deals with an arbitrary angle of incidence and with the general case where the reflected and transmitted fields may contain components whose propagation direction is not parallel to the incident wave.

Consider wave scattering by a grating of $N$ identical rows of cylinders as described at the 
beginning of $\S 6$; the configuration is assumed to be symmetric about $x=0$. A wave with potential

$$
\phi_{I}=\mathrm{e}^{\mathrm{i} \kappa r \cos \left(\theta-\theta_{q}\right)}
$$

is incident from the left at an angle $\theta_{q}$ to the $x$ axis, where $(r, \theta)$ are polar coordinates defined by $(x, y)=(r \cos \theta, r \sin \theta)$. This wave will be diffracted to obtain a reflected field

$$
\phi \sim \sum_{p=-\mu}^{\nu} R_{q p} \mathrm{e}^{-\mathrm{i} \kappa r \cos \left(\theta+\theta_{p}\right)} \quad \text { as } \quad x \rightarrow-\infty
$$

and a transmitted field

$$
\phi \sim \sum_{p=-\mu}^{\nu} T_{q p} \mathrm{e}^{\mathrm{i} \kappa r \cos \left(\theta-\theta_{p}\right)} \quad \text { as } \quad x \rightarrow \infty
$$

where

$$
\begin{gathered}
\sin \theta_{p}=\sin \theta_{q}+\frac{2 p \pi}{\kappa W}, \\
\mu=\left[\left(1+\sin \theta_{q}\right) \kappa W / 2 \pi\right] \text { and } \nu=\left[\left(1-\sin \theta_{q}\right) \kappa W / 2 \pi\right]
\end{gathered}
$$

and [.] indicates that the integer part should be taken (see Twersky, 1962). The far-field forms (70)(71) are valid for any integer $q \in[-\mu, \nu]$. Conservation of energy requires that the components of the reflection and transmission matrices $R$ and $T$ satisfy

$$
\sum_{p=-\mu}^{\nu}\left(\left|R_{q p}\right|^{2}+\left|T_{q p}\right|^{2}\right) \cos \theta_{p}=\cos \theta_{q}
$$

(Twersky, 1962). An algorithm for the computation of approximations to the reflection and transmission matrices for multiple rows from the properties of a single row is given by Heckl \& Mulholland $(1995, \S 3.2)$, and their formulation was used for the calculations described below. The reflection and transmission matrices for a single row were calculated using the method of Linton \& Evans (1993).

Heckl \& Mulholland (1995) also give an approximate method for the calculation of the Bloch transmission coefficient. When compared with the present methods there is good agreement except in the vicinity of $\kappa W=2 n \pi$, for positive integer $n$, where there can be considerable disagreement.

Computations for scattering by multiple rows are now used to illustrate the relationship between that problem and the Bloch problem of wave transmission through an infinite array. The results in figure 10 are for normal incidence $\left(\theta_{q}=0\right)$ and show how the quantity

$$
E_{T}=\sum_{p=-\mu}^{\nu}\left|T_{q p}\right|^{2} \cos \theta_{p},
$$

which is proportional to the transmitted wave energy, varies with the number of rows $N$. Results are given for three wave numbers $\kappa L \in(2 \pi, 4 \pi)$ for which, according to (73), there are a total of 


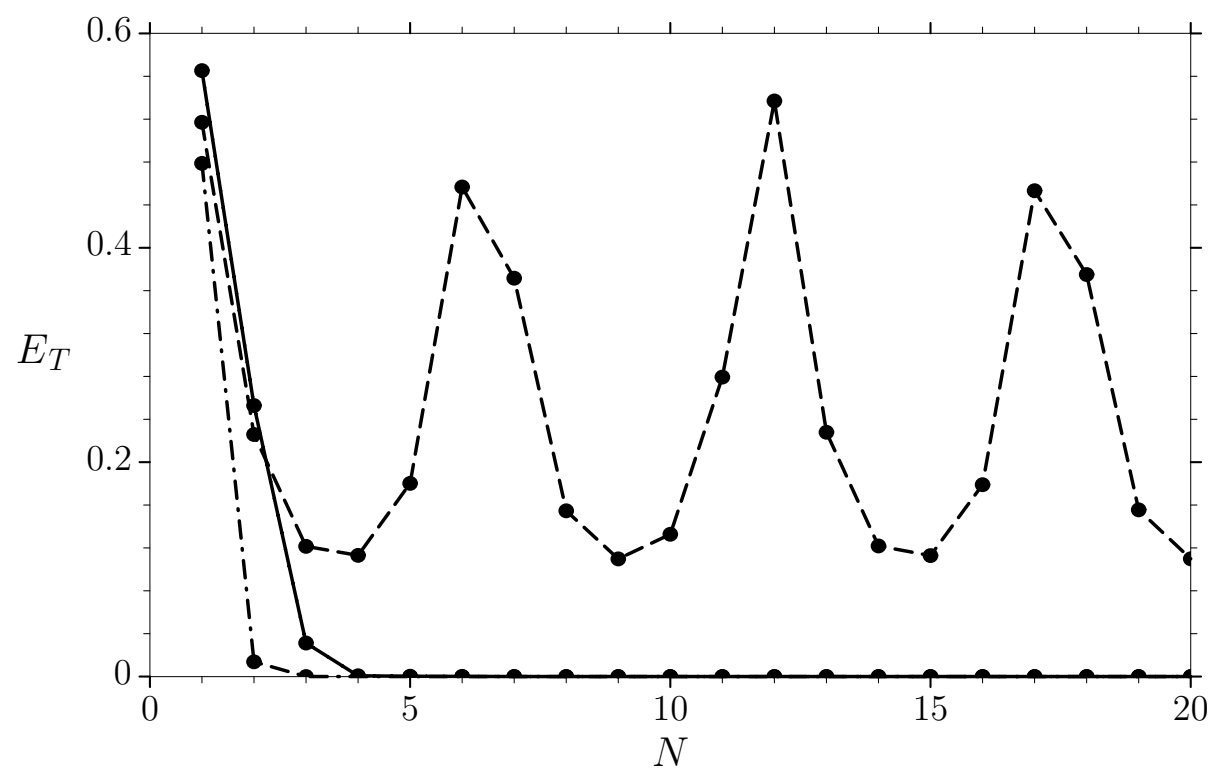

Figure 10: Transmitted energy $E_{T}$ for normal wave incidence $v s$. number of rows of cylinders $N ; W / L=1, D / L=0.5, \kappa L=2.7 \pi(-\cdot-\cdot-), \kappa L=3 \pi\left(--^{-}\right)$, $\kappa L=3.7 \pi(-)$.

three propagating wave directions. The wavenumber $\kappa L=2.7 \pi$ is within a stopping band (see figure 4) and there is very little energy transmission through more than two rows of cylinders. By contrast, $\kappa L=3 \pi$ is within a passing band and there is significant wave transmission through any number of rows. The third value $\kappa L=3.7 \pi$ is not within a stopping band. However, it can be seen from figure 4 that the only mode that can propagate through an infinite array is antisymmetric about $y=0$. However, under normal incidence, the scattering problem is completely symmetric and antisymmetric waves cannot be excited leading to suppression of wave transmission through the finite array. If the angle of incidence is not zero but instead one of the other associated propagation directions, so that $\sin \theta_{q}=2 \pi / \kappa W$ say, then the problem is longer completely symmetric and there is significant energy transmission through any number of rows for $\kappa L=3.7 \pi$. However, the transmission in the 'symmetric' normal direction is still negligible.

In this scattering problem, the complete solution has the form

$$
\phi(x, y)=\mathrm{e}^{\mathrm{i} q_{2} y} \psi(x, y)
$$

where

$$
q_{2}=\kappa \sin \theta_{q}
$$

and $\psi$ is periodic in $y$ with period $W$. Thus, the $y$ variation is exactly that occurring in the Bloch problem. By specifying $q_{2}$ in the Bloch problem according to equation (77) and solving for the 


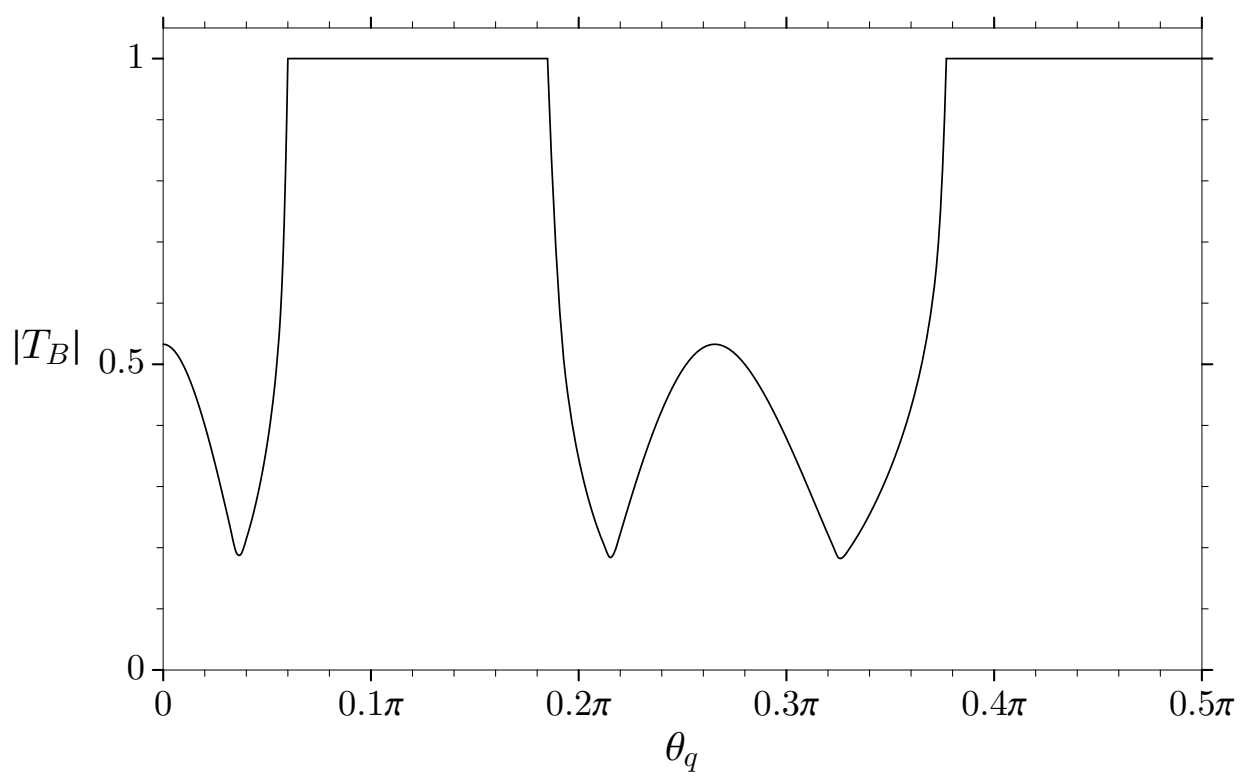

Figure 11: Maximum Bloch transmission $\left|T_{\mathrm{B}}\right|$ vs. angle of incidence $\theta_{q} ; W / L=1$, $D / L=0.5, \kappa L=2.7 \pi$.

Bloch transmission coefficient $T_{\mathrm{B}}$ useful information can be obtained about the scattering problem. For most wave numbers there are at least two distinct values of $\left|T_{\mathrm{B}}\right|$ and hence, in figure 11 , the maximum $\left|T_{\mathrm{B}}\right|$ is plotted as a function of angle for fixed $\kappa L$. The variational method was used to identify the passing bands and the boundary-integral method used to make calculations within the stopping bands. When $\left|T_{\mathrm{B}}\right|=1$ significant wave energy can be expected to propagate through a finite number of rows. When $\left|T_{\mathrm{B}}\right|<1$ little wave energy will propagate through the complete system and the decay with distance can be estimated from $\left|T_{\mathrm{B}}\right|$.

A wider picture in figure 12 shows the distribution of passing and stopping regions as a function of incidence angle and wave number. This figure was constructed using the technique described at the end of $\S 4.1$. In the unshaded regions, $\max \left\{\left|T_{\mathrm{B}}\right|\right\}=1$ and in general there will be significant wave transmission through a finite array. In the shaded regions $\left|T_{\mathrm{B}}\right|<1$ and, in general, wave transmission will be effectively blocked within the first few rows of a finite array.

\section{Conclusion}

A variety of techniques have been presented for the calculation of water-wave propagation through an doubly-periodic infinite array of vertical cylinders extending throughout the fluid depth. The phenomena of stopping and passing bands that arise in solid-state physics have been shown to arise in this water-wave Bloch problem as well. 


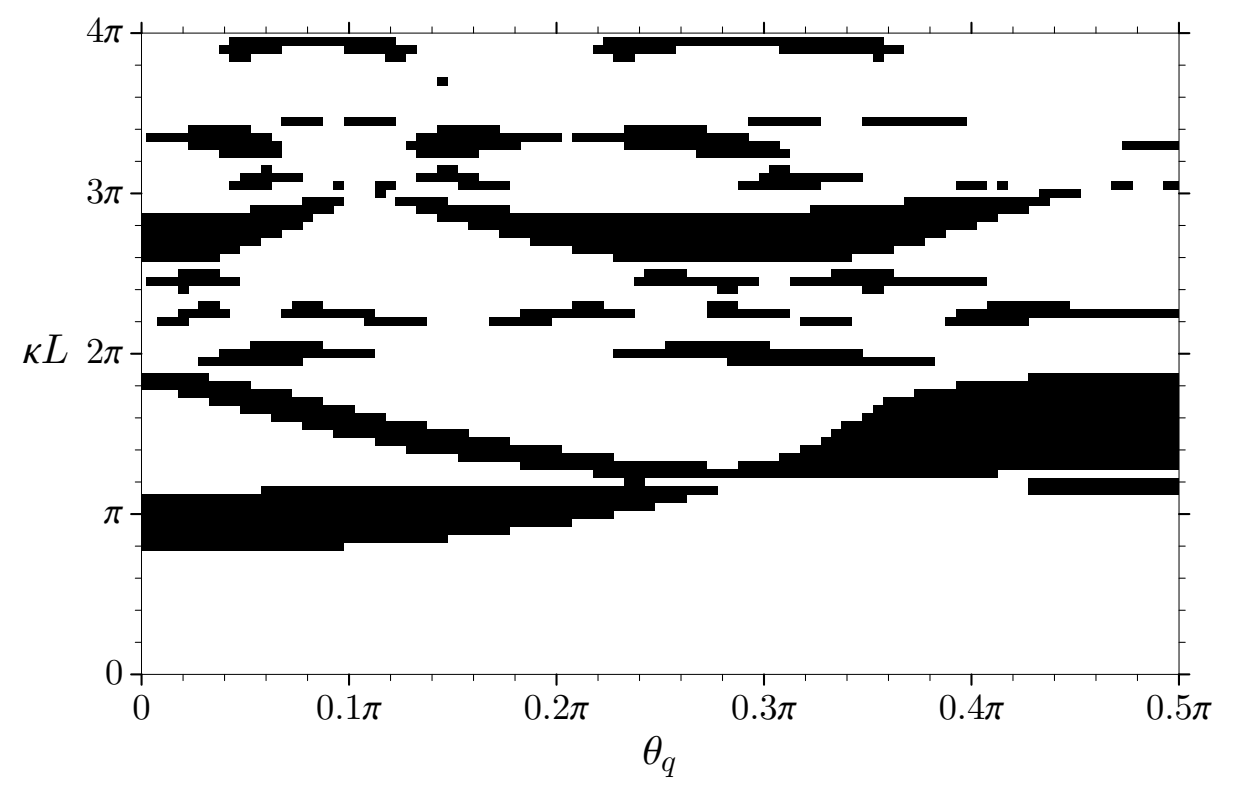

Figure 12: Passing (unshaded) and stopping (shaded) regions as a function of angle of incidence $\theta_{q}$ and wave number $\kappa L ; W / L=1, D / L=0.5$.

The point-matching technique of $\S 5.1$ may be extended to other geometries by modifying the series in equation (47). For example, the corresponding series for a circular dock can be calculated using the method given by Garrett (1971). For this geometry, the potential will no longer have the form (1). However, (1) will hold approximately sufficiently far from the cylinder for evanescent modes to be negligible, and so an approximate theory strictly valid only for small cylinder to spacing ratios is obtained. A similar modification of the Green's function (50) can be made in the boundary-integral method of $\S 5.2$.

The final sections of the paper are devoted to the relationship between the Bloch problem and water-wave scattering by a finite number of rows, but where each row is of infinite extent. Further work is required to relate the Bloch problem to wave scattering by an array that is finite in both horizontal directions.

\section{References}

The author is grateful to Dr. C. M. Linton for providing reflection and transmission data based on the work of Linton \& Evans (1993). 


\section{References}

Abramowitz, M. \& Stegun, I. A. 1964 Handbook of Mathematical Functions. National Bureau of Standards, Washington.

Ashcroft, N. W. \& Mermin, N. D. 1976 Solid State Physics. Saunders College, Philadelphia.

Callan, M., Linton, C. M. \& Evans, D. V. 1991 Trapped modes in two-dimensional wave guides. Journal of Fluid Mechanics, 229, 51-64.

Chou, T. 1998 Band structure of surface flexural-gravity waves along periodic interfaces. Journal of Fluid Mechanics, 369, 333-350.

Duff, G. F. C. \& Naylor, D. 1966 Differential Equations of Applied Mathematics. John Wiley \& Sons.

Evans, D. V. 1990 The wide-spacing approximation applied to multiple scattering and sloshing problems. Journal of Fluid Mechanics, 210, 647-658.

Evans, D. V. \& Porter, R. 1999 Trapping and near-trapping by arrays of cylinders in waves. Journal of Engineering Mathematics, 35, 149-179.

Garrett, C. J. R. 1971 Wave forces on a circular dock. Journal of Fluid Mechanics, 46, 129-139.

Golub, G. H. \& Van Loan, C. F. 1983 Matrix Computations. The Johns Hopkins University Press, Baltimore.

Heckl, M. A. 1992 Sound propagation in bundles of periodically arranged cylindrical tubes. Acustica, 77, 143-152.

Heckl, M. A. \& Mulholland, L. S. 1995 Some recent developments in the theory of acoustic transmission in tube bundles. Journal of Sound and Vibration, 179, 37-62.

Kagemoto, H. 1998 Wave decay characteristics along a long array of cylindrical legs. Proceedings of the 13th International Workshop on Water Waves and Floating Bodies, Alphen aan den Rijn, The Netherlands, 55-58. Delft University of Technology, The Netherlands.

Kagemoto, H. \& Yue, D. K. P. 1986 Wave forces on a platform supported on a large number of floating legs. Proceedings of the 5th International Conference on Offshore Mechanics and Arctic Engineering, 1, 206-211. ASME. 
Kashiwagi, M. 1999 A hierarchical interaction theory for wave forces on a great number of buoyancy bodies. Proceedings of the 14th International Workshop on Water Waves and Floating Bodies, Port Huron, Michigan, USA, 68-71. University of Michigan, USA.

Linton, C. M. \& Evans, D. V. 1993 The interaction of waves with a row of circular cylinders. Journal of Fluid Mechanics, 251, 687-708.

Maniar, H. \& Newman, J. N. 1997 Wave diffraction by long arrays of cylinders. Journal of Fluid Mechanics, 339, 309-330.

McIver, P., Linton, C.M. \& McIver, M. 1998 Construction of trapped modes for wave guides and diffraction gratings. Proceedings of the Royal Society of London A, 454, 2593-2616.

Mei, C. C. 1983 The Applied Dynamics of Ocean Surface Waves. John Wiley \& Sons.

O'Hare, T. J. \& Davies, A. G. 1993 A comparison of two models for surface-wave propagation over rapidly varying topography. Applied Ocean Research, 15, 1-11.

Porter, R. \& Evans, D. V. 1999 Rayleigh-Bloch surface waves along periodic gratings and their connection with trapped modes in waveguides. Journal of Fluid Mechanics, 386, 233-258.

Twersky, V. 1962 On scattering of waves by the infinite grating of circular cylinders. IRE Transactions on Antennas and Propagation, 10, 737-765. 